\title{
Neutrinos from Tidal Disruption Events
}

\section{Cecilia Lunardini*}

Department of Physics, Arizona State University, 450 E. Tyler Mall, Tempe, AZ 85287-1504 USA

E-mail: Cecilia.Lunardini@asu.edu

When a star is disrupted by a supermassive black hole, and its debris are accreted onto it, a powerful hadronic jet can be generated and be a source of high energy neutrinos and cosmic rays. I review the simplest models of neutrino production from such Tidal Disruption Events (TDEs), and show that the resulting neutrino flux is detectable, and can explain a fraction of the diffuse neutrino flux detected at IceCube. The possibility of a joint explanation of the neutrino and UltraHigh Energy Cosmic Ray data is briefly illustrated. I discuss the hypothesis - which requires further study to be fully substantiated - that a single TDE burst might have been responsible for the 2014-2015 neutrino flare observed by IceCube.

36th International Cosmic Ray Conference -ICRC2019-

July 24th - August 1st, 2019

Madison, WI, U.S.A.

${ }^{*}$ Speaker. 


\section{Introduction: tidal disruptions as particle accelerators}

When a star wanders too close to a supermassive black hole (SMBH), it can be torn into pieces and ultimately accreted onto the black hole. As a result of such Tidal Disruption Event (TDE), a flare of radiation in the thermal, UV and X-rays is produced [1, 2, 3, 4], lasting for days, months or even years. TDEs are very interesting in astronomy because they allow to probe black holes that are usually quiet (in contrast with the active galactic nuclei, AGN), and therefore difficult to study. Tens of candidate TDEs have been observed, and more frequent observations are expected with near-future surveys (see, e.g., [5]).

Interestingly, some TDE observations carry signatures of a relativistic jet in their X-ray emission. The best studied of such jetted TDEs is Swift J1644+57 [6]. The idea of TDEs as the cause of relativistic jets - probably with hadronic content, from the matter of the disrupted star - leads to considering them a possible source of Ultra-High Energy Cosmic Rays (UHECR) [7], and, as byproduct of the latter via photo-hadronic interaction, of high energy neutrinos $[8,9,10]$.

Recently, realistic, detailed studies have been performed on high energy neutrinos from TDEs, with focus on their possible contribution to the observed diffuse neutrino flux at IceCube [11, 12, 13]. The possibility of a joint interpretation of the neutrino data and of the UHECR data - with attention to the heavy nuclear composition of the cosmic rays - has also been studied [14, 15]. In the current theoretical context, where the the interpretation of the IceCube data is still open to several hypotheses, and at the same time multi-messenger constraints are already placing tight constraints on the historically favored interpretations (starburst galaxies, AGN, gamma ray bursts, etc., see, e.g., [16] and references therein), TDEs have emerged as an attractive alternative, for their being dark in $\mathrm{GeV}-\mathrm{TeV}$ gamma-rays, and for their characteristically long duration. Both such features weaken the constraints on TDE neutrino emission, allowing TDEs to account for a large fraction of the IceCube observed flux.

In Sec. 2 I will review the theory of neutrinos from TDEs, following mainly Refs. [13, 14]. In Sec. 3 new, preliminary, material [17] will be presented, proposing the hypothesis that the TXS $0506+056$ neutrino historical flare [18] could be due to a TDE.

\section{Neutrinos from Tidal Disruption Events: single burst and diffuse flux}

\subsection{Basic TDE physics and rates}

Consider a star of mass $m$ and radius $R$ (with $m$ and $R$ roughly of solar size, $m=M_{\odot} \simeq$ $1.99 \times 10^{33} \mathrm{~g}$ and $R=R_{\odot} \simeq 6.96 \times 10^{10} \mathrm{~cm}$ ), moving on a path that approaches a SMBH, of mass $M$. Let us also consider the ideal scenario of a black hole in vacuum (no prior accretion activity, etc.). As the star becomes closer to the SMBH, it can be deformed, and ultimately destroyed by tidal forces. Basic arguments of balancing of forces $[1,2,3,4]$ show that this happens when the star's distance to the SMBH falls below the tidal radius: $r_{t}=(2 M / m)^{1 / 3} R \simeq 8.8 \times$ $10^{12} \mathrm{~cm}\left(M / 10^{6} M_{\odot}\right)^{1 / 3}\left(R / R_{\odot}\right)\left(m / M_{\odot}\right)^{-1 / 3}$, if this radius is larger than the SMBH Schwarzschild radius, $r_{t}>R_{s}=2 M G / c^{2} \simeq 3 \times 10^{11} \mathrm{~cm}\left(M / 10^{6} M_{\odot}\right)$. The latter condition implies that disruption can occur if $M<M_{\max } \simeq 10^{7.2} M_{\odot}$ [19]. After disruption, about $\sim 1 / 2$ of the mass of the star becomes bound to the SMBH and is ultimately accreted on it. Therefore, a total energy $E_{\max } \sim M_{\odot} c^{2} / 2 \simeq 9 \times 10^{53} \mathrm{erg}$ is available for the jet. If the mass infall rate is sufficiently high 
a jet can be generated, with super-Eddington luminosity that declines with time as $\sim t^{-5 / 3}$ [2]. It is estimated that a fraction $\eta \sim 0.1$ of all TDEs host such jets [6]. The flare will last as long as the infall rate remains above the Eddington rate, $\Delta T \sim \mathscr{O}(0.1-1)$ yr [2]. Therefore, TDEs are relatively long transients, very different from second-long phenomena like the Gamma-Ray Bursts.

The cosmological rate of TDEs is very uncertain. The predicted rate per SMBH is $\dot{N}_{\mathrm{TD}} \simeq$ $10^{-3.7}\left(M / 10^{6} M_{\odot}\right)^{-0.1} \mathrm{yr}^{-1}[19]$. Combining it with the SMBH mass function, which scales roughly like $M^{-3 / 2}$ [20], gives the volumetric (comoving) rate, differential in $M, \dot{\rho}(z, M) \propto M^{-1.6}(1+$ $z)^{-3}$, and the total rate $R(z)=\int_{M_{\min }}^{M_{\max }} \dot{\rho}(z, M) d M$. A major uncertainty is the lower cutoff mass, $M_{\min }$, of the SMBH for which disruption can be effective (see, e.g. [20]). One gets a local rate of $R(0) \sim 810^{-6} \mathrm{Mpc}^{-3} \mathrm{yr}^{-1}$ for $M_{\text {min }}=10^{5} M_{\odot}\left(R(0) \sim 10^{-6} \mathrm{Mpc}^{-3} \mathrm{yr}^{-1}\right.$ for $\left.M_{\text {min }}=10^{6} M_{\odot}\right)$. Considering the jet beaming angle, and the fraction $\eta$, the rate of observable jetted TDEs is expected to be $\tilde{R}(0) \simeq 0.35-10 \mathrm{Gpc}^{-3} \mathrm{yr}^{-1}$, depending on $M_{\text {min }}$.

\subsection{Neutrino production in a TDE jet}

As a phenomenological approach, one can consider photohadronic neutrino production in a generic jet, with parameters set to match those of the best observed TDE, Swift J1644+57. These values will appear in the equations below and in Table 1 (with exceptions, see Table caption). The starting elements of the neutrino flux calculation are the background photon (X-ray) spectrum and the proton spectrum, which are taken as having power-law forms (spectral indices in Table 1).

Table 1: Parameters of the model for the neutrino fluence in fig. 3, in the SMBH frame. All the parameters are set to reproduce those of the Swift J1644+57 TDE, except $t_{v}$ and $\Gamma$ (which scale with $M$ ). See [13].

\begin{tabular}{|c|lr|}
\hline Symbol & Definition & value \\
\hline$t_{v}$ & Variability timescale & $6.3 \mathrm{~s}$ \\
$\Gamma$ & Lorentz factor & 6 \\
$\xi_{p}$ & Baryonic loading (energy in protons versus X-rays) & 10 \\
$\xi_{B}$ & Magnetic loading (energy in magnetic field versus X-rays) & 1 \\
$k_{p}$ & Proton spectral index & 2 \\
$E_{X}$ & Isotropic equivalent energy in X-rays & $3 \cdot 10^{53} \mathrm{erg}$ \\
$\Delta T$ & Duration of X-ray flare & $10^{6} \mathrm{~s}$ \\
$\varepsilon_{X, \mathrm{br}}$ & Observed X-ray break energy & $1 \mathrm{keV}$ \\
$\alpha$ & Lower X-ray spectral index $\varepsilon<\varepsilon_{X, \mathrm{br}}$ & $2 / 3$ \\
$\beta$ & Higher X-ray spectral index $\varepsilon>\varepsilon_{X, \mathrm{br}}$ & 2 \\
\hline
\end{tabular}

Although a numerical approach is necessary for precision, it is useful to introduce some approximate analytical scalings [8]. The fluence of muon neutrinos can be expressed as $E^{2} F_{\mu}^{0}(E) \propto$ $E_{X} \xi_{p} f_{p \gamma} \zeta_{\pi}\left(1+\zeta_{\mu}\right)$. Here the total energy in protons is expressed as proportional to the energy in $\mathrm{X}$-rays, $E_{p}=E_{X} \xi_{p}$, via the phenomenological constant $\xi_{p}$. The quantity $f_{p \gamma}$ is the pion production 
efficiency, i.e., the average fraction of energy deposited into pion production:

$f_{p \gamma} \simeq 0.35\left(\frac{L_{X}}{10^{47.5} \mathrm{erg} \mathrm{s}^{-1}}\right)\left(\frac{\Gamma}{10}\right)^{-4}\left(\frac{t_{v}}{10^{2} \mathrm{~s}}\right)^{-1}\left(\frac{\varepsilon_{b}}{\mathrm{KeV}}\right)^{-1} \times\left\{\begin{array}{l}\left(E_{p} / E_{p b}\right)^{\beta-1} \text { for } E_{p}<E_{p, \mathrm{br}} \\ \left(E_{p} / E_{p b}\right)^{\alpha-1} \text { for } E_{p} \geq E_{p, \mathrm{br}}\end{array}\right.$.

Here $L_{X}=E_{X} / \Delta T$ is the average $\mathrm{X}$-ray luminosity, and $E_{p, \text { br }}$ is the proton energy leading to photo-pion production at the $\Delta$-resonance corresponding to the X-ray break energy, i.e., $E_{p \text {,br }}=$ $1.510^{7} \mathrm{GeV}(\Gamma / 10)^{2}\left(1 \mathrm{KeV} / \varepsilon_{X, \mathrm{br}}\right) . \Gamma$ is the jet bulk Lorentz factor and $t_{v}$ its minimum variability time scale (which influences the collision radius $R_{c}$ where shell collisions take place, see [13]). The factors $\zeta_{\pi}$ and $\zeta_{\mu}$ are energy-dependent suppression factors that account for pion and muon propagation (energy losses and decay).

Naturally, the jet parameters will vary with the type of SMBH and star involved in the disruption. In [13], some tentative parameter scalings with $M$ were proposed. Here I review the "strong scaling" scenario, where $t_{v}$ is determined by the SMBH Schwarzschild time (a relationship that was suggested in the interpretation of the Swift J1644+57 data, [6]): $t_{v} \simeq \tau_{s}$, with $\tau_{s} \equiv$ $2 \pi R_{s} / c \simeq 63 \mathrm{~s}\left(M / 10^{6} M_{\odot}\right)$. Moreover, $\Gamma$ scales in a way that was found to fit AGN data [21]: $\Gamma(M)=\left(M / 10 M_{\odot}\right)^{0.2}$. All other parameters are constants, as in Table 1. The detailed simulation of the neutrino production and propagation was performed with the NeuCosmA code [22]. Fig. 1 (left panel) shows the predicted muon neutrino fluences for different values of $M$. The strong enhancement of the neutrino emission for decreasing $M$ is explained by how the scalings of $t_{v}$ and $\Gamma$ influence the pion production efficiency, Eq. (2.1).
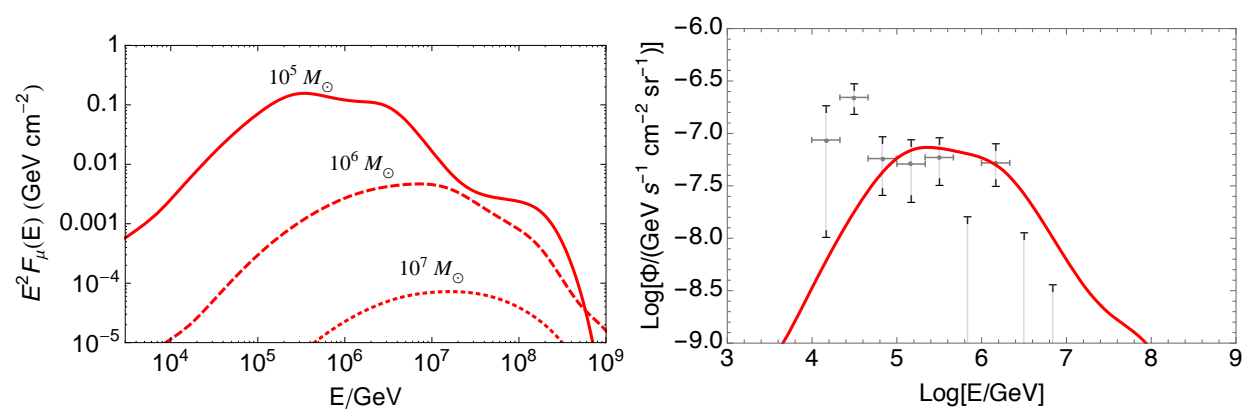

Figure 1: Left: from [13]: the fluence of $v_{\mu}+\bar{v}_{\mu}$, as a function of the neutrino energy, for a single TDE at $z=0.35$, in the strong scaling model, for different SMBH masses. Right: Adapted from [13]: the corresponding diffuse flux of $v_{\mu}+\bar{v}_{\mu}$, for $M_{\min }=10^{5} M_{\odot}$. The product $G=\xi_{p} \times \eta$ has been set to $G=0.2$ (below the reference value, $G=1$ ) to reproduce the measured IceCube flux at $E \simeq \mathrm{PeV}$ (shown, from [23, 24]).

\subsection{Diffuse flux and connection with the UHECR}

The diffuse flux of neutrinos is obtained by convolving the neutrino emission of a single TDE with the cosmological rate of TDEs, with inclusion of neutrino oscillations and redshift effects. Because the rate $\dot{\rho}(z, M)$ declines with $M$ (Sec. 2.1), the diffuse flux decreases rapidly as $M_{\min }$ increases, and is strongly affected by the uncertainty on this parameter. Fig. 1 (right panel), shows the diffuse flux predicted by the strong scaling model, with $M_{\min }=10^{5} M_{\odot}$, compared with the measured IceCube flux after 6 years of data taking (taken from [23, 24]). In the figure, the product $G=\xi_{p} \times \eta$ has been adjusted to a more conservative value (see caption) to match the data. This 
adjustment implies that the current IceCube data already constrain the interesting region of the parameter space of the model. From the figure, one can also see that the model reproduces the observed energy spectrum well above $\sim 100 \mathrm{TeV}$.

Motivated in part by the success of TDE models on the neutrino front, applications to the UHECR have been studied. In the latter context, TDEs are attractive as a potential source of medium-heavy nuclei $[25,26,14,15]$, which could explain the relatively heavy composition of the UHECR seen at the Pierre Auger Observatory [28, 27]. The most natural scenario is the disruption of carbon-oxygen white dwarfs, which is can be realized efficiently for intermediate mass black holes $\left(M \sim\left(10^{3}-10^{5}\right) M_{\odot}\right)$. In [14], the production and propagation of neutrinos and UHECR was
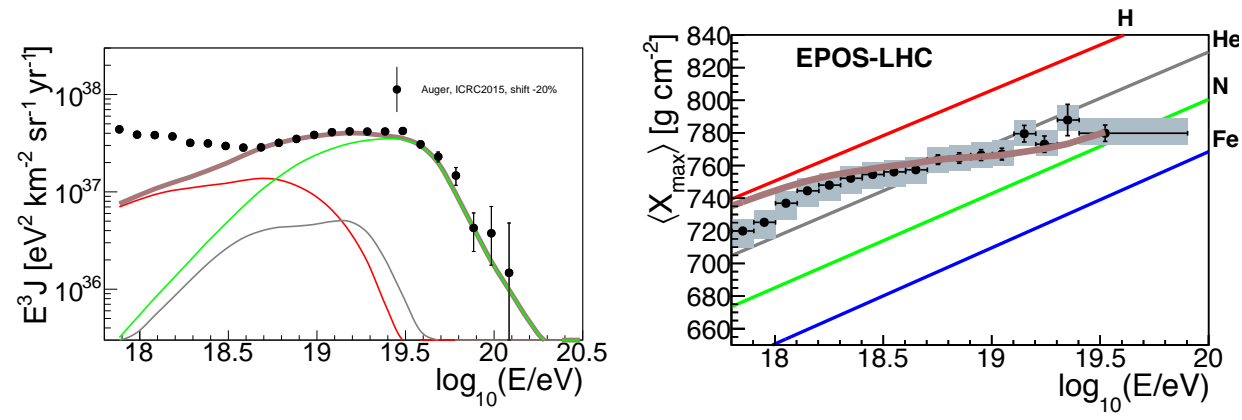

Figure 2: Taken from [14]. Left: Simulated energy spectrum of UHECRs (thick); and its components from (groups of) different nuclear species (thin, color coding in right panel). The Auger data are shown [28]. Right: Predictions and data [27] on the average of the $X_{\max }$ distributions as a function of the energy.

simulated consistently, by including the effects of nuclear cascading in the jet as well as cosmic ray propagation in the intergalactic medium. For simplicity, a single nuclear species, ${ }^{14} \mathrm{~N}$, was injected in the jet, as (accurate) approximation of the mixed carbon-oxygen injection. The numerical codes and methodology used are those in [22, 29, 30]. An example of resulting UHECR spectrum and composition is shown in Fig. 2; the agreement with the data is very good. The corresponding neutrino flux matches the observed neutrino spectrum at IceCube at $\sim \mathrm{PeV}$ energy (see [14]). Thus, it is possible that the entire, diverse TDE phenomenology, including both white dwarf and main sequence stars, might account for most of the UHECR and neutrino data.

\section{The TXS 0506+056 historical flare: a tidal disruption?}

An analysis of IceCube archival data at the coordinates of the blazar TXS 0506+056 (TXS from here on, redshift $z=0.336$ ) revealed the likely detection of a flare of $13 \pm 5$ neutrino events in 2014-2015 [18], with duration $\Delta T \simeq 110 \pm 20$ days, total fluence is $\Phi_{100}=2.1_{-0.7}^{+0.9} 10^{-1} \mathrm{GeV} \mathrm{cm}^{-2}$, and estimated isotropic equivalent luminosity $L_{V} \sim 1.2 \times 10^{47} \mathrm{ergs} / \mathrm{s}$. Electromagnetic observations of TXS for the same time period are sparse, and available data are consistent with the timeaveraged blazar emission (see [31, 32], and references therein).

The three month-long duration of the neutrino flare immediately suggests a TDE as a natural explanation, corroborated by the relatively high energy of the event $\left(L_{v} \times \Delta T \sim 1.610^{54}\right.$ ergs isotropic equivalent energy, suggestive of a disrupted star of a few solar masses, see Sec. 2.1). Intriguingly, the strong scaling TDE model discussed above, with $M_{\min }=10^{5} M_{\odot}$, reproduces the measured fluence and spectrum very well (without any fitting to the data), as shown in Fig. 3 
(left panel). The TDE interpretation becomes more complex, however, once the multi-messenger
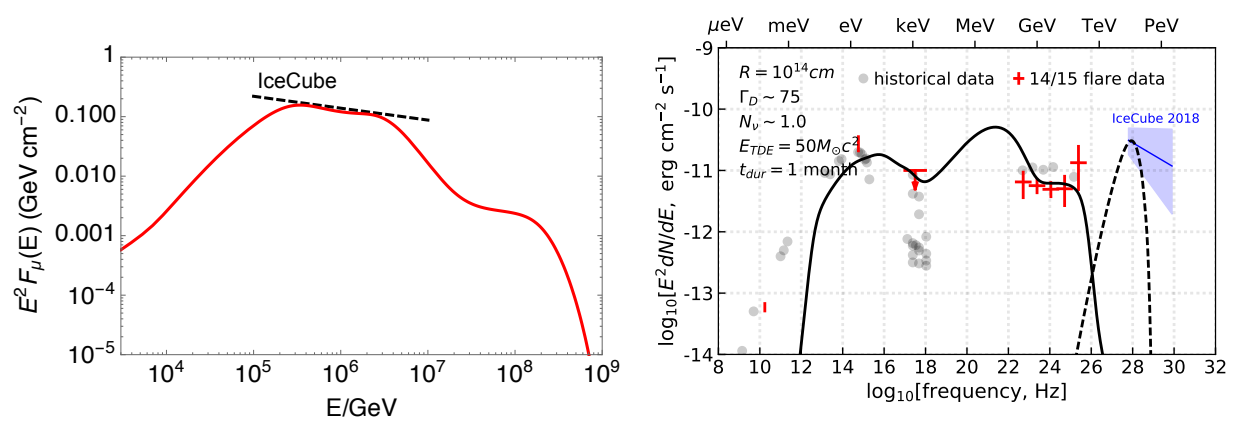

Figure 3: Spectra for the TXS historical flare. Left: The measured neutrino fluence (dashed), and the corresponding prediction for the $M=10^{5} M_{\odot}$ strong scaling model (solid; same as fig. 1; see Table 1 for the parameters). The good agreement is accidental. Right: Multimessenger plot, showing the photon spectrum data, the neutrino flare spectrum, and a prediction in the compact core model (solid: photons; dashed: neutrinos. Figure courtesy of Shan Gao, based on [33]).

picture of the flare is considered. Indeed, preliminary calculations [17] show that in the strong scaling model the predicted photon flux exceeds the measurements and upper bounds synchronous to the flare. This is known to be a general problem of a minimal jet framework, see, e.g. [34, 33]. Another issue is that the strong scaling model (and the TDE interpretation in general) is in tension with the high estimated mass of the TXS SMBH $\left(M_{T X S} \simeq 310^{8} M_{\odot}\right.$ [35]), which exceeds $M_{\max }$ (Sec. 2.1) and would require a variability time scale much greater than that in Table 1.

To improve the TDE interpretation of the historical flare, one can turn to non-minimal jet models. The fact that TXS is an active galaxy, with a pre-existing jet and accretion disk, is a motivation to use a compact core model (see, e.g., [33]), where the jet is made of two zones, one wider and with lower particle/radiation density (the pre-existing jet), which can explain the low energy part of the photon spectrum, and the other more compact and denser (the core, due to the TDE), which is responsible for the high energy gamma rays and neutrinos. Unfortunately, the compact model is found to be unable to accommodate all the observations: it can reproduce the neutrino spectrum and exceed photon constraints, or accommodate the latter at the price of underpredicting the number of neutrino events in the flare. Fig. 3 (right panel) shows the latter case, where the electromagnetic constraints are saturated and only one neutrino event is predicted (note also the unnaturally high energy budget, corresponding to the disruption of a $\sim 50 M_{\odot}$ star). These results agree with those in [33].

Despite the issues, a TDE interpretation of the historical flare is still a possibility. It might require including other potentially important phenomena. For example, the accretion torus generated by the debris of the disrupted star could cause significant absorption effects, and possibly choke a jet $[9,12]$. Absorption might partially explain why Swift J1644+57 was dark in gamma rays above $100 \mathrm{MeV}$ [6]. Indications of significant absorption of X-rays, consistent with an accretion disk, have been found in the (non-jetted) TDE PS16dtm [36]. It is also conceivable that an undetected black hole with $M \sim 10^{5} M_{\odot}$ might exist in the vicinity of the core of TXS, and disrupt a Sun-like star, with possible effects due to the interplay with the central black hole and its accretion disk. 


\section{References}

[1] J. G. Hills, Possible power source of seyfert galaxies and qsos, Nature 254 (03, 1975) 295-298.

[2] M. J. Rees, Tidal disruption of stars by black holes of 10 to the 6th-10 to the 8th solar masses in nearby galaxies, Nature 333, 523 (1988).

[3] J. H. Lacy, C. H. Townes, and D. J. Hollenbach, The nature of the central parsec of the Galaxy, Astrophys. J. 262 (Nov., 1982) 120-134.

[4] E. S. Phinney, Manifestations of a Massive Black Hole in the Galactic Center, in The Center of the Galaxy (M. Morris, ed.), vol. 136 of IAU Symposium, p. 543, 1989.

[5] S. Komossa, Tidal disruption of stars by supermassive black holes: Status of observations, JHEAp 7, 148 (2015) [arXiv:1505.01093].

[6] D. N. Burrows et al., Discovery of the Onset of Rapid Accretion by a Dormant Massive Black Hole, Nature 476, 421 (2011) [arXiv: 1104 .4787].

[7] G. R. Farrar and A. Gruzinov, Giant AGN Flares and Cosmic Ray Bursts, Astrophys. J. 693, 329 (2009) [arXiv:0802.1074].

[8] X. Y. Wang, R. Y. Liu, Z. G. Dai and K. S. Cheng, Probing the tidal disruption flares of massive black holes with high-energy neutrinos, Phys. Rev. D 84,081301 (2011) [arXiv:1106.2426].

[9] X. Y. Wang and R. Y. Liu, Tidal disruption jets of supermassive black holes as hidden sources of cosmic rays: explaining the IceCube TeV-PeV neutrinos, Phys. Rev. D 93, no. 8, 083005 (2016) [arXiv:1512.08596].

[10] K. Murase and K. Ioka, TeV?PeV Neutrinos from Low-Power Gamma-Ray Burst Jets inside Stars, Phys. Rev. Lett. 111, no. 12, 121102 (2013) [arXiv: 1306.2274].

[11] L. Dai and K. Fang, Can tidal disruption events produce the IceCube neutrinos?, Mon. Not. Roy. Astron. Soc. 469, no. 2, 1354 (2017) [arXiv: 1612 . 00011 ].

[12] N. Senno, K. Murase and P. Meszaros, High-energy Neutrino Flares from X-Ray Bright and Dark Tidal Disruption Events, Astrophys. J. 838, no. 1, 3 (2017) [arXiv:1612.00918].

[13] C. Lunardini and W. Winter, High Energy Neutrinos from the Tidal Disruption of Stars, Phys. Rev. D 95, no. 12, 123001 (2017) [arXiv:1612.03160].

[14] D. Biehl, D. Boncioli, C. Lunardini and W. Winter, Tidally disrupted stars as a possible origin of both cosmic rays and neutrinos at the highest energies, Sci. Rep. 8, no. 1, 10828 (2018) [arXiv:1711.03555].

[15] C. Guépin, K. Kotera, E. Barausse, K. Fang and K. Murase, Ultra-High Energy Cosmic Rays and Neutrinos from Tidal Disruptions by Massive Black Holes, Astron. Astrophys. 616, A179 (2018) [arXiv:1711.11274].

[16] M. Ahlers and F. Halzen, Opening a New Window onto the Universe with IceCube, Prog. Part. Nucl. Phys. 102, 73 (2018) [arXiv: 1805.11112].

[17] S. Gao, C. Lunardini, A. Palladino and W. Winter, work in progress.

[18] M. G. Aartsen et al., Neutrino emission from the direction of the blazar TXS 0506+056 prior to the IceCube-170922A alert, Science 361, no. 6398, 147 (2018) [arXiv:1807.08794].

[19] C. S. Kochanek, Tidal disruption event demographics, Mon. Not. Roy. Astron. Soc. 461, no. 1, 371 (2016) [arXiv: 1601.06787$].$ 
[20] F. Shankar, D. H. Weinberg and J. Miralda-Escude, Self-Consistent Models of the AGN and Black Hole Populations: Duty Cycles, Accretion Rates, and the Mean Radiative Efficiency, Astrophys. J. 690, 20 (2009) [arXiv:0710.4488].

[21] B. Chai, X. Cao and M. Gu, What governs the bulk velocity of the jet components in active galactic nuclei?, Astrophys. J. 759, 114 (2012) [arXiv:1209.4702].

[22] D. Biehl, D. Boncioli, A. Fedynitch and W. Winter, Cosmic-Ray and Neutrino Emission from Gamma-Ray Bursts with a Nuclear Cascade, Astron. Astrophys. 611, A101 (2018) [arXiv:1705.08909].

[23] M. G. Aartsen et al., The IceCube Neutrino Observatory - Contributions to ICRC 2015 Part II: Atmospheric and Astrophysical Diffuse Neutrino Searches of All Flavors, [arXiv: 1510 . 05223].

[24] M. G. Aartsen et al., A combined maximum-likelihood analysis of the high-energy astrophysical neutrino flux measured with IceCube, Astrophys. J. 809, no. 1, 98 (2015) [arXiv: 1507.03991 ].

[25] R. Alves Batista and J. Silk, Ultrahigh-energy cosmic rays from tidally-ignited white dwarfs, Phys. Rev. D 96, no. 10, 103003 (2017) [arXiv: 1702 .06978].

[26] B. T. Zhang, K. Murase, F. Oikonomou and Z. Li, High-energy cosmic ray nuclei from tidal disruption events: Origin, survival, and implications, Phys. Rev. D 96, no. 6, 063007 (2017) [arXiv:1706.00391].

[27] A. Porcelli, for the Pierre Auger Collaboration, Measurements of the first two moments of the depth of shower maximum over nearly three decades of energy, combining data from, PoS(ICRC2015)420, 2015.

[28] I. Valino, for the Pierre Auger Collaboration, The flux of ultra-high energy cosmic rays after ten years of operation of the Pierre Auger Observatory, PoS(ICRC2015)271, 2015.

[29] R. Aloisio, D. Boncioli, A. Di Matteo, A. F. Grillo, S. Petrera and F. Salamida, SimProp v2r4: Monte Carlo simulation code for UHECR propagation, JCAP 1711, no. 11, 009 (2017) [arXiv:1705.03729].

[30] P. Baerwald, M. Bustamante and W. Winter, UHECR escape mechanisms for protons and neutrons from GRBs, and the cosmic ray-neutrino connection, Astrophys. J. 768, 186 (2013) [arXiv:1301.6163].

[31] S. Garrappa et al., Investigation of two Fermi-LAT gamma-ray blazars coincident with high-energy neutrinos detected by IceCube, [arXi v:1901.10806].

[32] P. Padovani, P. Giommi, E. Resconi, T. Glauch, B. Arsioli, N. Sahakyan and M. Huber, Dissecting the region around IceCube-170922A: the blazar TXS 0506+056 as the first cosmic neutrino source, Mon. Not. Roy. Astron. Soc. 480, no. 1, 192 (2018) [arXiv: 1807.04461 ].

[33] X. Rodrigues, S. Gao, A. Fedynitch, A. Palladino and W. Winter, Leptohadronic Blazar Models Applied to the 2014?2015 Flare of TXS 0506+056, Astrophys. J. 874, no. 2, L29 (2019) [arXiv: 1812.05939].

[34] S. Gao, A. Fedynitch, W. Winter and M. Pohl, Modelling the coincident observation of a high-energy neutrino and a bright blazar flare, Nat. Astron. 3, no. 1, 88 (2019) [arXiv: 1807.04275 ].

[35] P. Padovani, F. Oikonomou, M. Petropoulou, P. Giommi and E. Resconi, TXS 0506+056, the first cosmic neutrino source, is not a BL Lac, Mon. Not. Roy. Astron. Soc. 484, no. 1, L104 (2019) [arXiv:1901.06998].

[36] P. K. Blanchard et al., PS16dtm: A Tidal Disruption Event in a Narrow-line Seyfert 1 Galaxy, Astrophys. J. 843, no. 2, 106 (2017) [arXiv: 1703.07816 ]. 